\title{
Efectos teratogénicos de insecticidas organofosforados en la etiología de labio y paladar hendido: revisión de literatura
}

Jesús Gabriel Ortega-Miller, JG, Od ${ }_{1}$, Salomón Yezioro Rubinsky ${ }^{\star}$, Esp $_{1}$, Berta Cecilia Benavides-Pinto, Msc. ${ }_{2}$, Liliana Carolina Báez-Quintero, Msc. ${ }_{3}$

\author{
${ }_{1}$ Facultad de Odontología, Universidad Nacional de Colombia, Bogotá, Colombia \\ , Universidad Cooperativa de Colombia, Bogotá, Colombia \\ ${ }_{3}$ Universidad Estadual Paulista, Câmpus de Araçatuba, São Paulo, Brasil
}

Recibido: 23 de marzo del 2016 Aprobado: 15 de agosto del 2016

*Autor de correspondencia: Salomón Yezioro, Facultad de Odontología, Oficina de correspondencia, Facultad de Odontología, piso 3, oficina Torres Pinzón, Universidad Nacional de Colombia, sede Bogotá. Correo electrónico: syezioror@unal.edu.co

Cómo citar este artículo: Ortega-Miller JG, Yezioro-Rubinsky S, Benavides-Pinto BC, Báez-Quintero LC. Efectos teratogénicos de insecticidas organofosforados en la etiología de labio y paladar hendido: revisión de literatura. Rev Nac Odontol. 2017;13(24):101-110. doi: http://dx.doi.org/10.16925/od.v12i24.1658

Resumen. Introducción: el objetivo fue revisar la evidencia científica sobre la asociación entre la exposición de individuos a insecticidas organofosforados, con la prevalencia de labio y paladar fisurado no sindrómico. Métodos: se realizaron búsquedas de artículos científicos en las bases de datos Pubmed, Science Direct, Google Académico, Medline, Cochrane y Scielo, publicados entre el 2000 y el 2015. Se buscaron artículos teniendo en cuenta los términos MESH, incluyendo estudios de revisión de literatura, metanálisis, casos y controles, y experimentales in vivo, excluyéndose estudios experimentales in vitro. Se encontraron 25 artículos que cumplieron con los criterios de inclusión. Resultados: un significativo número de estudios establecieron una asociación entre la exposición a insecticidas órganofosforados con efectos teratogénicos relacionados con la formación del labio y paladar hendido; estudios experimentales in vivo en animales relacionaron la exposición al insecticida organofosforado clorpirifós, con mayor prevalencia de paladar hendido. Conclusiones: los resultados de la presente revisión de literatura no establecen asociación entre la exposición a insecticidas organofosforados y efectos teratogénicos relacionados con la formación de labio y paladar hendido.

Palabras clave: anomalías craneofaciales, agroquímicos, fisura del paladar, insecticidas, insecticidas organofosforados, labio leporino. 


\title{
Teratogenic effects of organophosphate insecticides on cleft lip and palate etiology: A literature review
}

\begin{abstract}
Introduction: The objective was to review the scientific evidence on the association between exposure of individuals to organophosphate insecticides and the prevalence of non-syndromic cleft lip and palate. Methods: Scientific articles published between 2000 and 2015 were searched on Pubmed, Science Direct, Google Scholar, Medline, Cochrane and Scielo databases. Articles were searched considering the MESH terms, including literature review, meta-analyses, cases and controls, and in vivo experimental studies, excluding in vitro experimental studies. Twenty-five articles that met the inclusion criteria were found. Results: A significant number of studies established an association between exposure to organophosphate insecticides and teratogenic effects related to cleft lip and palate formation; in vivo experimental studies on animals related exposure to the chlorpyrifos organophosphorus insecticide to a higher prevalence of cleft palate. Conclusions: The results of the present literature review do not establish an association between exposure to organophosphate insecticides and teratogenic effects related to cleft lip and palate formation.
\end{abstract}

Keywords: craniofacial anomalies, agrochemicals, cleft palate, insecticides, organophosphate insecticides, cleft lip.

\section{Efeitos teratogênicos de inseticidas organofosforados na etiologia de lábio e palato fendidos: revisão de literatura}

Resumo. Introdução: o objetivo foi revisar a evidência científica sobre a associação entre a exposição de indivíduos a inseticidas organofosforados, com a prevalência de lábio e palato fissurados não sindrômicos. Métodos: foram realizadas pesquisas de artigos científicos nas bases de dados Pubmed, Science Direct, Google Acadêmico, Medline, Cochrane e Scielo, publicados entre 2000 e 2015. Buscaram-se artigos levando em consideração os termos MESH, incluindo estudos de revisão de literatura, meta-análise, casos e controles, e experimentais in vivo, excluindo estudos experimentais in vitro. Foram encontrados 25 artigos que cumpriram com os critérios de inclusão. Resultados: um número significativo de estudos estabeleceu uma associação entre a exposição a inseticidas organofosforados com efeitos teratogênicos relacionados com a formação do lábio e palato fendidos; estudos experimentais in vivo em animais relacionaram a exposição ao inseticida organofosforado clorpirifós com maior prevalência de palato fendido. Conclusões: os resultados da presente revisão de literatura não estabelecem associação entre a exposição a inseticidas organofosforados e efeitos teratogênicos relacionados com a formação de lábio e palato fendidos.

Palavras-chave: anomalias craniofaciais, agroquímicos, fissura do palato, inseticidas, inseticidas organofosforados, lábio leporino. 


\section{Introducción}

Las fisuras labio palatinas no sindrómicas (FLPNS) son malformaciones cráneo faciales de frecuencia diversa según localización geográfica, raza y estatus socioeconómico. Su etiología es compleja y multifactorial, en la cual confluyen factores genéticos, principalmente asociados a los genes Msx1, IRF6, TGFß3, TGFa, FGFR1, PVRL1 y al PAX9 [1-4]; a factores ambientales, entre los que se mencionan alcoholismo [5, 6], tabaquismo [7, 8], deficiencia de ácido fólico [9-11], fármacos antiepilépticos (fenitoína, ácido valproico) [11-13]; y a la exposición a pesticidas [13, 14], entre otros. La población afectada presenta alteraciones en su crecimiento y desarrollo cráneo facial, así como alta prevalencia de anomalías dentales, comparada con la población general. Por lo tanto, requiere de atención por parte del sistema de salud y ser visto como un problema de salud pública [15-17].

Diversos estudios en animales y humanos evidencian que la exposición materna a insecticidas organofosforados aumenta el riesgo de presentar defectos del tubo neural y del sistema nervioso, anencefalia, espina bífida, labio y/o paladar hendido, reducciones de las extremidades y muerte [18-20].

La identificación de factores de riesgo asociados con la etiología de fisuras orales constituye el primer paso hacia su prevención, lo cual implica modificaciones en el estilo de vida materna y paterna, mejora de la dieta, restricción y/o control del acceso y manipulación de ciertas drogas y medicamentos, y el conocimiento general de los factores de riesgo sociales, ocupacionales y residenciales [21].

La presente revisión de literatura tiene como propósito revisar la información sobre la asociación entre la exposición de individuos a insecticidas organofosforados con la prevalencia de FLPNS en la literatura científica.

\section{Materiales y métodos}

Se realizó una búsqueda de artículos en las bases de datos Pubmed, ScienceDirect, Google
Académico, Medline, Cochrane y Scielo. Para la búsqueda se utilizaron las siguientes combinaciones de términos MEsh: "Cleft Palate or Cleft Lip OR Orofacial Cleft oR Craniofacial Anomalies AND Pesticides oR Agrochemicals or Insecticides OR Organophosphates". Se incluyeron revisiones bibliográficas y estudios llevados a cabo en humanos y animales, publicados entre enero del 2000 y diciembre del 2015, los cuales se presentaran en inglés, español y portugués, y relacionaran la exposición a insecticidas de uso agrícola con el desarrollo embriológico de anomalías congénitas, en particular, labio y/o paladar hendido. Se excluyeron artículos en los que no fue posible el acceso al texto completo por encontrarse en idiomas diferentes a los establecidos dentro de los criterios de inclusión, o que no se relacionaran directamente con el objetivo del estudio. La selección de los artículos que fueron incluidos en la revisión se realizó en tres fases: en la primera, se observó que el título del artículo correspondiera con el tema de revisión; en la segunda, se analizó el resumen teniendo en cuenta que el objetivo del estudio se relacionara con los propósitos de la revisión; en la tercera fase se evaluó el artículo en su totalidad (figura 1). Adicionalmente, se identificaron otros estudios usando las referencias bibliográficas de los artículos ya seleccionados, con el fin de incluir otros artículos no hallados durante la búsqueda en bases de datos.

\section{Resultados}

Finalmente, fueron seleccionados 28 artículos que cumplieron con los criterios de inclusión, 26 de los cuales se presentaron en idioma inglés, y dos en español. Tres de los estudios fueron revisiones de literatura, un metaanálisis, doce estudios de casos y controles, tres fueron estudios ecológicos y nueve experimentales in vivo en animales (véase la tabla 1). 


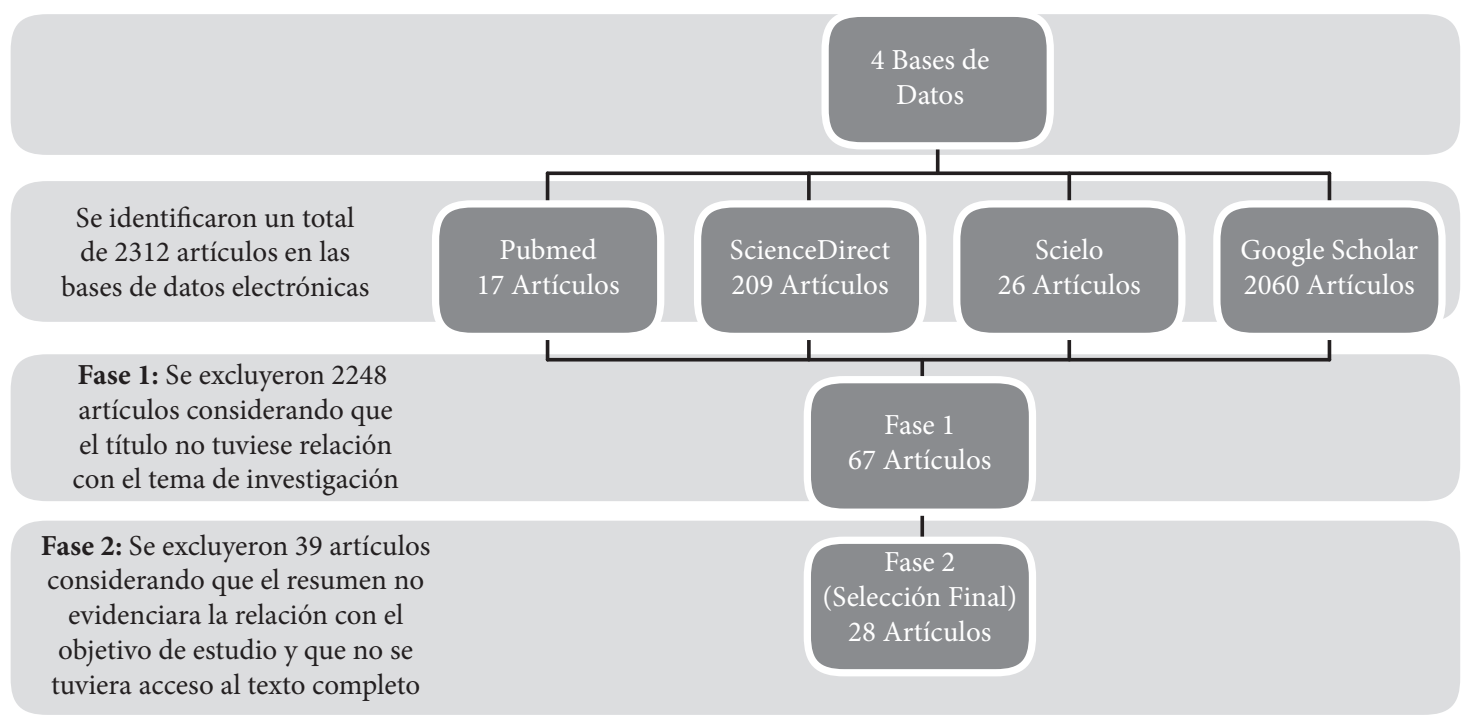

Figura 1. Mecanismo de selección de los artículos en cada fase de la revisión narrativa

Fuente: elaboración propia

Tabla 1. Artículos seleccionados en la revisión

\begin{tabular}{|c|c|c|c|}
\hline Título & Autor-Año & Diseño & Agente indagado \\
\hline Meta analysis: pesticides and orofacial clefts. & Romitti P (2007) & Metaanálisis & Pesticidas \\
\hline $\begin{array}{l}\text { The risk of adverse reproductive and developmental } \\
\text { disorders due to occupational pesticide exposure: an } \\
\text { overview of current epidemiological evidence. }\end{array}$ & Hanke W (2004) & Revisión de literatura & Pesticidas \\
\hline $\begin{array}{l}\text { Maternal occupational exposure and risk of specific } \\
\text { birth defects. }\end{array}$ & $\begin{array}{l}\text { Thulstrup AM } \\
\text { (2006) }\end{array}$ & Revisión de literatura & Pesticidas \\
\hline $\begin{array}{l}\text { Reproductive disorders associated with pesticides } \\
\text { expourse. }\end{array}$ & Frazier LM (2008) & Revisión de literatura & Pesticidas \\
\hline $\begin{array}{l}\text { A study of the impact of agricultural pesticide use on the } \\
\text { prevalence of birth defects in northeast Italy. }\end{array}$ & Clementi M (2007) & Ecológico & $\begin{array}{l}\text { Pesticidas y otros factores de } \\
\text { riesgo. }\end{array}$ \\
\hline $\begin{array}{l}\text { Oral clefts a restrospective study of prevalence and } \\
\text { predisposal factor in the stade of Mexico. }\end{array}$ & González BS (2008) & Ecológico & $\begin{array}{l}\text { Pesticidas y otros factores de } \\
\text { riesgo. }\end{array}$ \\
\hline $\begin{array}{l}\text { Correlation between pesticide use in agriculture and } \\
\text { adverse birth outcomes in Brazil: an ecological study. }\end{array}$ & Teixeira M (2010) & Ecológico & Pesticidas \\
\hline $\begin{array}{l}\text { Maternal occupational chemical exposures and bio- } \\
\text { transformation genotypes as risk factors for selected } \\
\text { congenital anomalies. }\end{array}$ & Shaw GM (2003) & Casos y controles & $\begin{array}{l}\text { Pesticidas y otros factores de } \\
\text { riesgo. }\end{array}$ \\
\hline $\begin{array}{l}\text { Orofacial clefts in the newborn and environmental and } \\
\text { occupational parental exposures: a case-control study in } \\
\text { Rio de Janeiro Brazil. }\end{array}$ & Leite IC. (2003) & Casos y controles & $\begin{array}{l}\text { Insecticidas de uso en hoga- } \\
\text { res, pesticidas de uso agrícola } \\
\text { y fumigación urbana. }\end{array}$ \\
\hline $\begin{array}{l}\text { Parental exposures and risk of nonsyndromic orofacial } \\
\text { clefts in offspring: A case-control study in Greece. }\end{array}$ & Mirilas P (2011) & Casos y controles & $\begin{array}{l}\text { Pesticidas y otros factores de } \\
\text { riesgo. }\end{array}$ \\
\hline $\begin{array}{l}\text { Pre- and post-conception pesticide exposure and the } \\
\text { risk of birth defects in an Ontario farm population. }\end{array}$ & Weselak M (2008) & Casos y controles & Pesticidas. \\
\hline $\begin{array}{l}\text { Paternal occupational exposures and the risk of congeni- } \\
\text { tal malformations a case-control study. }\end{array}$ & EL-Helaly M (2011) & Casos y controles- & $\begin{array}{l}\text { Pesticidas y otros factores de } \\
\text { riesgo. }\end{array}$ \\
\hline Congenital malformations and pesticide exposure. & Rojas A (2000) & Casos y controles & Pesticidas. \\
\hline
\end{tabular}


(viene)

\begin{tabular}{|c|c|c|c|}
\hline Título & Autor-Año & Diseño & Agente indagado \\
\hline $\begin{array}{l}\text { Ocupational exposure to pesticides and pregnancy } \\
\text { outcomes in gardeners and farmers: a study within the } \\
\text { danish national birth cohort. }\end{array}$ & Zhu J L (2006) & Casos y controles & Pesticidas. \\
\hline $\begin{array}{l}\text { Parental pesticidal exposure and risk of clefts in the } \\
\text { craniofacial region: a case-control study in south India. }\end{array}$ & Betty J (2015) & Casos y controles & Pesticidas. \\
\hline $\begin{array}{l}\text { Residential agricultural pesticide exposures and risk of } \\
\text { neural tube defects and orofacial clefts among offspring } \\
\text { in the San Joaquin Valley of California. }\end{array}$ & Yang W (2014) & Casos y controles & Pesticidas. \\
\hline $\begin{array}{l}\text { Factores ambientales asociados con labio o paladar } \\
\text { hendido no sindrómico en una población del magdalena } \\
\text { medio colombiano. }\end{array}$ & Prada AM (2014) & Casos y controles & $\begin{array}{l}\text { Pesticidas y otros factores de } \\
\text { riesgo. }\end{array}$ \\
\hline $\begin{array}{l}\text { Association of Parental Environmental Exposures and } \\
\text { Supplementation Intake with Risk of Nonsyndromic } \\
\text { Orofacial Clefts: A Case-Control Study in Heilongjiang } \\
\text { Province, China. }\end{array}$ & Hao Y (2015) & Casos y controles & $\begin{array}{l}\text { Pesticidas y otros factores de } \\
\text { riesgo. }\end{array}$ \\
\hline $\begin{array}{l}\text { A Case-control Study of Environmental Risk Factors for } \\
\text { Nonsyndromic Cleft of the Lip and/or Palate in Xuzhou, } \\
\text { China. }\end{array}$ & Xu LF (2015) & Casos y controles & $\begin{array}{l}\text { Pesticidas y otros factores de } \\
\text { riesgo. }\end{array}$ \\
\hline Developmental toxicity of acephate by gavage in mice. & Farag AT (2000) & Experimental & $\begin{array}{l}\text { Insecticida organofosforado } \\
\text { - Acefato. }\end{array}$ \\
\hline Developmental toxicity study of chlorpyrifos in rats. & Farag AT (2003) & Experimental & $\begin{array}{l}\text { Insecticida organofosforado - } \\
\text { Clorpirifos. }\end{array}$ \\
\hline $\begin{array}{l}\text { Teratogenicity and developmental toxicity of chlorpyri- } \\
\text { fos: Maternal exposure during organogenesis in mice. }\end{array}$ & Tian Y (2005) & Experimental & $\begin{array}{l}\text { Insecticida organofosforado - } \\
\text { Clorpirifos. }\end{array}$ \\
\hline $\begin{array}{l}\text { Histological assessment of the teratogenic potential of } \\
\text { the pesticide Folidol to induce the appearance of cleft } \\
\text { palate in wistar rats. }\end{array}$ & $\begin{array}{l}\text { Camargo E S. } \\
(2006)\end{array}$ & Experimental & $\begin{array}{l}\text { Insecticida organofosforado } \\
\text { - Folidol. }\end{array}$ \\
\hline $\begin{array}{l}\text { The Effects of Trichlorfon on Maternal Reproduction } \\
\text { and Mouse Embryo Development during Organoge- } \\
\text { nesis. }\end{array}$ & Tian Y (2009) & Experimental & $\begin{array}{l}\text { Insecticida organofosfora- } \\
\text { do-Trichlorfon. }\end{array}$ \\
\hline $\begin{array}{l}\text { Oral exposure of male and female mice to formulations } \\
\text { of rganophosphorous pesticides: congenital malforma- } \\
\text { tions. }\end{array}$ & Gomes J (2008) & Experimental & $\begin{array}{l}\text { Mezcla de Insecticidas Orga- } \\
\text { nofosforados. }\end{array}$ \\
\hline $\begin{array}{l}\text { Developmental toxicity studies in rats and rabbits with } \\
\text { 3,5,6-trichloro-2-pyridinol, the major metabolite of } \\
\text { chlorpyrifos. }\end{array}$ & Hanley TR (2000) & Experimental & $\begin{array}{l}\text { Insecticida organofosforado } \\
\text { - TCP (principal metabolito } \\
\text { del Chlorpyrifos). }\end{array}$ \\
\hline $\begin{array}{l}\text { Transplacental disposition and teratogenic effects of } \\
\text { chlorpyrifos in rats. }\end{array}$ & Akhtar N (2006) & Experimental & $\begin{array}{l}\text { Insecticida organofosfora- } \\
\text { do-Clorpirifos. }\end{array}$ \\
\hline $\begin{array}{l}\text { Analysis of cytogenetic and developmental effects on } \\
\text { pre-implantation, mid-gestation and near-term mouse } \\
\text { embryos after treatment with trichlorfon during zygote } \\
\text { stage. }\end{array}$ & Tian Y (2000) & Experimental & $\begin{array}{l}\text { Insecticida organofosfora- } \\
\text { do-Triclorfon. }\end{array}$ \\
\hline
\end{tabular}

Fuente: elaboración propia

\section{Revisiones de literatura y metaanálisis}

Los estudios de revisión bibliográfica y metaanálisis evidenciaron un mayor riesgo teratogénico en la descendencia, por la exposición a pesticidas durante el período periconcepcional [22-24]. Estos reflejaron que la exposición parental a pesticidas puede aumentar el riesgo de anormalidades cualitativas y cuantitativas en producción de espermatozoides e infertilidad en ambos géneros $[22,24]$, muerte fetal, aborto espontaneo, retardo del crecimiento fetal, defectos de los sistemas musculares y esqueléticos $[22,24]$ y hendiduras orales [22]. Solo un autor afirmó que la evidencia encontrada hasta la fecha es limitada para determinar una conclusión [25]. 


\section{Estudios en humanos}

\section{Estudios ecológicos}

La mayoría de estudios ecológicos seleccionados en esta revisión evidenciaron la hipótesis según la cual los pesticidas están asociados con efectos teratogénicos en el embrión, ya sea por exposición materna, o bien sea por paterna [26, 27]. Un estudio no encontró asociación significativa entre la aparición de FLPNS y la exposición ocupacional paterna, pero sí una relación significativa entre las actividades agrícolas maternas y esta anomalía, sugiriendo que la exposición a pesticidas se asocia con alteraciones en el desarrollo de labio y/o paladar hendido [27]. Solo un autor propone que no hay asociación entre los pesticidas y efectos teratogénicos durante la embriogénesis [28].

\section{Estudios de casos y controles}

Existe controversia en los estudios revisados de casos y controles llevados a cabo en humanos. La mayoría evidenció asociación entre la exposición a pesticidas durante el período periconcepcional, y mayor riesgo de malformaciones congénitas [29-37], como labio y/o paladar hendido [29, 31, 32, 34-37], defectos cardiacos [31], y de los sistemas gastrointestinal, genitourinario y nervioso central [30] en los hijos de los padres expuestos. Adicionalmente, se encontró relación entre la exposición a pesticidas y parto prematuro [30]. Por el contrario, otros estudios no hallaron algún tipo de asociación significativa entre malformaciones congénitas y el trabajo en la agricultura o la exposición a pesticidas, incluyendo insecticidas organofosforados [38-40].

\section{Estudios experimentales in vivo}

Estos estudios realizados en animales han evaluado la relación entre la exposición a insecticidas organofosforados con el aumento de la prevalencia de paladar hendido, pero no con la de labio con o sin paladar hendido, entidades con diferente etiología. Sus resultados presentan alto grado de controversia; tres de ellos reportaron relación entre la exposición a insecticidas organofosforados y efectos teratogénicos en la formación del paladar $[18,19,41]$. La mayor controversia se relacionó con la exposición a clorpirifós; Farag, El Okazy y El-Aswed [19], así como Tian et al. [41], en el 2005, encontraron que la exposición de ratas y ratones a clorpirifós a dosis altas $(25 \mathrm{mg} / \mathrm{kg} /$ día y $80 \mathrm{mg} /$ $\mathrm{kg}$ dosis única, respectivamente), aumentó la incidencia de hendiduras palatinas; sin embargo, no sucedió esto en los especímenes que Tian et al. expusieron a $40 \mathrm{mg} / \mathrm{kg} / \mathrm{día}$ [41]. Contrariamente, Hanley no encontró aumento significativo en la incidencia de paladar hendido en ratas y conejos, relacionado con la exposición a dosis altas (50, 100, o $150 \mathrm{mg} / \mathrm{kg} / \mathrm{dí}$, y 25, 100, o $250 \mathrm{mg} / \mathrm{kg} /$ día, respectivamente) del químico 3,5,6-tricloro-2-piridinol (principal metabolito del clorpirifós) [42]. Además, Akhtar no reportó asociación entre la exposición a clorpirifós a dosis bajas y efectos teratogénicos en la formación del paladar en ratas [43]. Otros estudios tampoco hallaron relación entre la exposición a insecticidas organofosforados y efectos teratogénicos en la formación del paladar a dosis bajas [44-47], y altas [44-46], por vía oral $[45,46]$, tópica [47] o intraperitoneal [45], en animales. Los insecticidas organofosforados ejercen su acción tóxica al inactivar las enzimas acetilcolinesterasas, las cuales conforman un grupo de esterasas capaces de hidrolizar la acetilcolina (un neurotransmisor químico), y de esta forma se promueve la actividad colinérgica [42, 43]. Tian et al. consideran que los insecticidas organofosforados ejercen su efecto teratogénico en las fisuras palatinas al promover la actividad colinérgica en el feto durante el período de la embriogénesis, en la reorientación de las crestas palatinas por encima de la lengua, al plantear que el neurotransmisor serotonina influye en la reorientación de la parte anterior del paladar, mientras que la acetilcolina influye en la reorientación palatina posterior [41].

Adicionalmente, algunos estudios evidenciaron relación entre la exposición a insecticidas organofosforados durante la gestación, con el aumento de la incidencia de alteraciones tales como retraso del crecimiento intrauterino [18, 19], disminución del peso fetal [44], defectos del desarrollo en los oídos [18], los ojos [18, 19, 41], el cerebro [18], las extremidades [19, 44] y la pelvis [19].

\section{Discusión}

La presente revisión de literatura permite describir la literatura científica disponible sobre la asociación entre la exposición a pesticidas de uso agrícola, con un mayor riesgo de presentar labio y 
paladar hendido. Estudios experimentales en animales evidencian asociación entre la exposición a insecticidas organofosforados, con un mayor riesgo de presentar efectos teratogénicos en la formación del paladar [18, 19, 41]. Dos de estos estudios evidencian asociación entre la exposición a dosis altas al insecticida organofosforado clorpirifós, con un mayor riesgo de presentar paladar hendido [19, 41], mientras que otro no encontró esta asociación [42]. Este resultado contradictorio se podría explicar porque en este último estudio se realizó exposición solamente al TCP (metabolito primario del clorpirifós), y los efectos biológicos de clorpirifós se atribuyen principalmente a su biotransformación en el metabolito oxonclorpirifós, el cual actúa sinérgicamente para inhibir la acetilcolinesterasa [19].

Otros insecticidas organofosforados investigados son acefato, folidol y trichlorfon, a cuya exposición no se reportaron efectos teratogénicos en la formación del paladar [44-47]. Sin embargo, con la exposición a acefato y clorpirifós se encontró un aumento en la incidencia de otras alteraciones, como, por ejemplo, retraso del crecimiento intrauterino [18, 19], disminución del peso fetal [44], aumento de la incidencia de defectos del desarrollo en los oídos [18], los ojos [18, 19, 41], el cerebro [18], las extremidades [19, 44], y la pelvis [19]. El mecanismo de acción teratogénico de los insecticidas organofosforados no está aún bien definido.

Los estudios en humanos expuestos a pesticidas no especificaron el tipo utilizado (insecticidas, fungicidas, herbicidas, etc.), lo que dificultó concluir si la exposición a insecticidas de uso agrícola causa o no FLPNS.

Los resultados discrepantes entre los estudios en humanos se pueden atribuir a diferentes factores en las investigaciones, tales como: muestras pequeñas de la población o casos estudiados $[29,38,39]$; no considerar otros posibles factores etiológicos asociados con anomalías cráneo faciales como alcoholismo, tabaquismo e ingesta de medicamentos [32, 38]; la no utilización de medidas de protección a pesticidas por parte de la población estudiada [28]; sesgo de memoria de los padres en los estudios retrospectivos $[29,30]$; y diferencias en las dosis individuales y la frecuencia de exposición. Además, cabe contemplar que es difícil establecer los efectos causados por la exposición a cada uno de los diferentes tipos de pesticidas, ya que prácticamente en todos los casos los individuos se exponen de forma simultánea a varios, y el uso de estos varía de acuerdo con la temporada del año en que se den las cosechas o a la diversidad de plagas presentes [48].

En estudios en humanos realizados en Colombia, Restrepo se propuso como objetivo determinar la asociación entre la exposición ocupacional a pesticidas y la ocurrencia de malformaciones congénitas u otros defectos del nacimiento, encontrando un incremento de riesgo solo para marcas de nacimiento, específicamente hemangiomas, en niños con padres expuestos a los pesticidas [49]. Muñoz, por su parte, buscó establecer una asociación entre posibles factores de riesgo y algunas anomalías congénitas en la población colombiana, identificando el consumo de medicamentos durante la gestación y antecedentes de malformaciones familiares como factores de riesgo para LPH (no se incluyeron los pesticidas como posible factores de riesgo a evaluar) [50]. Prada buscó evaluar la posible asociación entre factores ambientales y LPH en niños residentes en el Magdalena Medio colombiano, encontrando una asociación estadísticamente significativa entre la exposición a pesticidas con el desarrollo de LPH (35).

La dosis, la vía de administración por la que se absorbe el agente y el momento de desarrollo embrionario en que ocurre la exposición al insecticida son factores causales determinantes [47], no considerados de manera rigurosa y común en todos los estudios que trataron de establecer la condición de teratogenicidad de estos productos, influyendo esto de manera relevante en las conclusiones de los estudios relacionados con los efectos teratogénicos del clorpirifós y otros insecticidas organofosforados evaluados. Adicionalmente, uno de los estudios señala que utilizaron dosis de acefato considerablemente más bajas que las reportadas, para generar efectos cancerígenos y mutagénicos [44], por lo cual queda abierta la duda de si esta sustancia puede aumentar la incidencia de FLPNS al exponer al huésped a dosis más altas.

Por otra parte, solo un estudio experimental utilizó más de una especie animal para la investigación [42], y una sola constitución genética. Es decir, una sola especie animal no es suficiente para evaluar la teratogenicidad de un agente [47], por lo que se recomienda probar los insecticidas en diversas especies antes de hacer afirmaciones acerca de su potencial teratogénico.

Los especialistas en farmacología, toxicología, genética, epidemiología, y en salud ocupacional y 
ambiental entrevistados, coinciden en que existe asociación entre la exposición a insecticidas durante la gestación con el aumento de la incidencia de FLPNS. Sin embargo, consideran difícil evidenciar el papel real de muchos de los pesticidas en la etiología de las FLPNS dados los escasos reportes encontrados en la literatura, así como por el hecho de que los protocolos de manejo de muchos pesticidas de uso agrícola en el país por parte de los agricultores incluyen la aplicación de combinaciones de varios tipos de productos al mismo tiempo, con el propósito teórico de atacar con mayor eficacia las plagas. Los controles se realizan sin que necesariamente exista una formulación profesional de agrónomos o profesionales relacionados con el control de plagas. Todos coinciden en la necesidad de reforzar los mecanismos de control para venta y uso de pesticidas en este país, ya que se ha encontrado la presencia de pesticidas prohibidos y altamente tóxicos en cultivos.

Se sugiere realizar nuevos estudios experimentales confiables en su desarrollo metodológico, controlando factores de sesgo tales como las condiciones de los modelos de estudio, el tipo de cepa, el medio de administrar las sustancias (tópico, oral, intraperitoneal), las dosis utilizadas y el tipo de exposición (crónica o aguda), a fin de llegar a conclusiones más contundentes sobre los efectos teratogénicos de los insecticidas en la formación del labio y el paladar.

Considerando que Colombia es un país exportador de productos agrícolas (flores, café, frutas), el uso de plaguicidas es frecuente. Por tanto, establecer los riesgos asociados a la etiología de FLPNS relacionados con insecticidas es relevante.

Se recomienda instaurar campañas de educación a los campesinos por parte del Estado Colombiano sobre el uso de estos productos, y mejorar los mecanismos de control, comercialización y uso de estos, ante la evidencia encontrada en los estudios publicados al respecto.

Este trabajo le aporta a la comunidad académica, a los trabajadores agrícolas y al personal relacionado con el uso de insecticidas, conocimientos sobre el riesgo generado con la exposición a estos durante el embarazo, particularmente durante el período de la formación del labio y el paladar. Pretende, igualmente, que las entidades de salud del Estado tomen medidas para el control de agroquímicos que puedan poner en riesgo la salud de los colombianos.

\section{Conclusiones}

Los resultados de la presente revisión de literatura no permitieron establecer asociación entre la exposición a insecticidas organofosforados y efectos teratogénicos relacionados con la formación del labio y paladar hendido.

La evidencia científica encontrada relaciona únicamente la exposición al insecticida organofosforado clorpirifós a dosis altas, con mayor prevalencia de paladar hendido en estudios in vivo en animales.

Se halló asociación entre la exposición a insecticidas organofosforados, con el aumento de la incidencia de alteraciones del nacimiento tales como retraso del crecimiento intrauterino, defectos de las extremidades y de los ojos.

Actualmente, no hay certidumbre acerca del mecanismo mediante el cual los insecticidas organofosforados originan efectos teratogénicos durante la formación del paladar.

\section{Referencias}

[1] Rahimov F, Jugessur A, Murray JC. Genetics of non syndromic orofacial clefts. Cleft Palate Cran J. 2012 Jan;49(1):73-91.

[2] Dixon MJ, Marazita ML, Beaty TH, Murray JC. Cleft lip and Palate: Understanding genetic and environmental influences. Nat Rev Genet. 2011 Mar; 12(3):167-78.

[3] Merritt L. Part 1 Understanding the Embryology and Genetics of Cleft Lip and Palate. Adv Neonatal Care. 2005 Apr; 5(2):64-71.

[4] Murray JC. Gene/environment causes of cleft lip and/or palate. Clin Genet.2002 Apr; 61(4):248-56.

[5] Lorente C, Cordier S, Goujard J, Ayme S, Bianchi $\mathrm{F}$, et al. Tobacco and alcohol use during pregnancy and risk of oral clefts. Am J Public Health. 2000; 90:415-19.

[6] Grewal J, Carmichael SL, Ma C, Lammer EJ, Shaw GM. Maternal periconceptional smoking and alcohol consumption and risk for select congenital anomalies. Birth Defects Res A Clin Mol Teratol. 2008 Jul;82(7):519-26.

[7] Li Z, Liu J, Ye R, Zhang L, Zheng X, Ren A. Maternal passive smoking and risk of cleft lip with or without cleft palate. Epidemiology. 2010 Mar;21(2):240-2.

[8] Chung K, Kowalski C, Kim H, Buchman S. Maternal Cigarette Smoking during Pregnancy and the Risk of Having a Child with Cleft Lip/Palate. Plastic \& Reconstructive Surgery. 2000 Feb;105(2):485-91. 
[9] Badovinac RL, Werler MM, Williams PL, Kelsey KT, Hayes C. Folic acid-containing supplement consumption during pregnancy and risk for oral clefts: A meta-analysis. Birth Defects Res A Clin Mol Teratol. 2007 Jan;79(1):8-15.

[10] Wilcox AJ, Lie RT, Solvoll K, Taylor J, McConnaughey DR, Abyholm F. Folic acid supplements and risk of facial clefts: National population based case-control study. BMJ 2007; 334 :464.

[11] Prabhu S, Krishnapillai R, Jose M, Prabhu V. Etiopathogenesis of orofacial clefting revisited. Journal of Oral and Maxillofacial Pathology. 2012;16 (2):228-232.

[12] Serrano C.A. Ruiz J.M. Quinceno L.F. Labio y o paladar hendido: una revisión. Revista UstaSalud. 2009:8:44-52.

[13] Bender PL. Genetics of cleft lip and palate. J Pediatr Nurs. 2000 Aug;15(4):242-9.

[14] Mirilas P, Mentessidou A, Kontis E, Asimakidou M, Moxhamb BJ, Petropoulos AS, et al. Parental exposures and risk of nonsyndromic orofacial clefts in offspring: A case-control study in Greece. Int J Pediatri Otorhinolaryngol. 2011 May;75(5):695-9.

[15] Bishara SE, de Arrendondo RS, Vales HP, Jakobsen JR. Dentofacial relationships in persons with unoperated clefts: Comparisons between three cleft types. Am J Orthod. 1985 Jun;87(6):481-507.

[16] Paranaiba LM, Coletta RD, Swerts MS, Quintino RP, de Barros LM, Martelli-Júnior H. Prevalence of Dental Anomalies in Patients With Nonsyndromic Cleft Lip and/or Palate in a Brazilian Population, Cleft Palate Craniofac J. 2013 Jul;50(4):400-5.

[17] Al Jamal GA, Hazza'a AM, Rawashdeh MA. Prevalence of dental anomalies in a population of cleft lip and palate patients. Cleft Palate Craniofac J. 2010 Jul;47(4):413-20.

[18] Gomes J, Lloyd OL and Hong Z. Oral exposure of male and female mice to formulations of organophosphorous pesticides: Congenital malformations. Hum Exp Toxicol. 2008 Mar; 27(3):231-40.

[19] Farag AT, El Okazy AM, El-Aswed AF. Developmental toxicity study of chlorpyrifos in rats. Reprod Toxicol. 2003 Mar-Apr;17(2):203-8.

[20] Lacasaña $M$, Vázquez-Grameix $H$, Borja-Aburto VH, Blanco-Muñoz J, Romieu I, Aguilar-Garduño $\mathrm{C}$, et al. Maternal and paternal occupational exposure to agricultural work and the risk of anencephaly. Occup Environ Med. 2006 Oct; 63(10):649-56.

[21] Mossey PA, Little J, Munger RG, Dixon MJ, Shaw WC. Cleft lip and Palate. The Lancet. 2009 Nov; 374(9703):1773-85.

[22] Hanke W, Jurewicz J. The risk of adverse reproductive and developmental disorders due to occupa- tional pesticide exposure: an overview of current epidemiological. Int J Occup Med Environ Health. 2004;17(2):223-43.

[23] Romitti PA, Herring AM, Dennis LK, Wong-Gibbons DL. Meta-analysis: Pesticides and orofacial clefts. Cleft Palate Craniofac J. 2007 Jul; 44(4):35865.

[24] Frazier LM. Reproductive disorders associated with pesticides expourse. J Agromedicine. 2007;12(1):2737.

[25] Thulstrup AM, Bonde JP. Maternal occupational exposure and risk of specific birth defects. Occupational Medicine. 2006;56:532-43.

[26] Teixeira M, Braga C, Cabral-Filho JE, Augusto LG, Figueiroa JN, Souza AI. Correlation between pesticide use in agriculture and adverse birth outcomes in Brazil. An ecological study. Bull Environ Contam Toxicol. 2010 Jun;84(6):647-51.

[27] González BS, López ML, Rico MA, Garduño F. Oral clefts a restrospective study of prevalence and predisposal factor in the state of Mexico. J Oral Sci. 2008 Jun;50(2):123-9.

[28] Clementi M, Causin R, Marzocchi C, Mantovani A, Tenconi R. A study of the impact of agricultural pesticide use on the prevalence of birth defects in northeast Italy. Reprod Toxicol. 2007 Jul; 24(1):1-8.

[29] Mirilas P, Mentessidou A, Kontis E, Asimakidou M, Moxhamb BJ, Petropoulos AS, et al. Parental exposures and risk of nonsyndromic orofacial clefts in offspring: A case-control study in Greece. Int J Pediatri Otorhinolaryngol. 2011 May; 75(5):695-9.

[30] El-Helaly M, Abdel-Elah K, Haussein A, Shalaby $H$. Paternal occupational exposures and the risk of congenital malformations a case control study. Int J Occup Med Environ Health. 2011 Jun; 24(2):218-27.

[31] Shaw GM, Nelson V, Iovannisci D, Finnell RH, Lammer E. Maternal Occupational Chemical Exposures and Biotransformation Genotypes as Risk Factors for Selected Congenital Anomalies. Am J Epidemiol. 2003 Mar;157(6):475-84.

[32] Leite IC, Roma Paumgartten FJ, Koifman S. Orofacial clefts in the newborn and environmental and occupational parental exposures: a case-control study in Rio de Janeiro, Brazil. Rev Bras Saude Mater Infant; 2003 Oct./Dec; 3(4):401-9.

[33] Rojas RAl, Ojeda BM, Barraza OX. Malformaciones congénitas y exposición a pesticidas. Rev Méd Chile. 2000 abr: 128(4):99-404.

[34] Betty J, Varsha M, Subramani S, Shashirekha M, Jayanthi K, Rajini T. Parental pesticidal exposure and risk of clefts in the craniofacial region: a case-control study in South India. International Journal of Applied Biology and Pharmaceutical Technology; 2015 June; 6(2); 230-35. 
[35] Prada AM, Eljach GM, Caballero V, Torres EA. Factores ambientales asociados con labio o paladar hendido no sindrómico en una población del magdalena medio colombiano. Rev. Ustasalud Odontología. 2014 ene;13(1):18- 25.

[36] Hao Y, Tian S, Jiao X, Mi N, Zhang B, Song T, et al. Association of parental environmental exposures and supplementation intake with risk of nonsyndromic orofacial clefts: A case-control study in Heilongjiang Province, China. Nutrients. 2015; 7(9):7172-84

[37] Xu LF, Zhou XL, Wang Q, Zhou JL, Liu YP, Ju Q, et al. A case-control study of environmental risk factors for nonsyndromic cleft of the lip and/or palate in Xuzhou, China. Biomed Environ Sci. 2015 jul;28(7): 535-8.

[38] Weselak M, Tye E. Arbuckle, Wigle DT, Walker MC, Krewsk Di. Pre- and post-conception pesticide exposure and the risk of birth defects in an Ontario farm population. Reprod Toxicol. 2008 Aug;25(4):472-80.

[39] Zhu JL, Hjollund NH, Andersen AM, Olsen J. Occupational exposure to pesticides and pregnancy outcomes in gardeners and farmers: A study within the Danish national birth cohort. J Occup Environ Med. 2006 Apr;48(4):347-52.

[40] Yang W, Carmichael SL, Roberts EM, Kegley SE, Padula AM, English PB, et al. Residential agricultural pesticide exposures and risk of neural tube defects and orofacial clefts among offspring in the San Joaquin Valley of California. Am J Epidemiol. 2014 Mar 15;179(6):740-8.

[41] Tian Y, Ishikawa H, Yamaguchi T, Yamauchi T, Yokoyama K. Teratogenicity and developmental toxicity of chlorpyrifos Maternal exposure during organogenesis in mice. Reprod Toxicol. 2005 JulAug;20(2):267-70.
[42] Hanley TR Jr, Carney EW, Johnson EM. Developmental toxicity studies in rats and rabbits with 3,5,6-trichloro-2-pyridinol, the major metabolite of chlorpyrifos. Toxicol Sci. 2000 Jan;53(1):100-8.

[43] Akhtar N, Srivastava M, Raizada R. Transplacental disposition and teratogenic effects of chlorpyrifos in rats. J Toxicol Sci. 2006 Dec; 31(5):521-7.

[44] Farag AT. Eweidahb MH, Tayelb SM, El-Sebaea AH. Developmental toxicity of acephate by gavage in mice. Reprod Toxicol. 2000 May-Jun;14(3):241-5.

[45] Tian Y, Ishikawa H, Yamauchi T. Analysis of cytogenetic and developmental effects on pre-implantation, mid-gestation and near-term mouse embryos after treatment with trichlorfon during zygote stage. Mutat Res. 2000 Nov 20;471(1-2):37-44.

[46] Tian Y, Dai F, Shen L, Feng Y, Gao Y, Xu L, et al. The effects of trichlorfon on maternal reproduction and mouse embryo development during organogenesis. Ind Health. 2009 Jul;47(3):313-8.

[47] Camargo ES, Vianna MS, Bolognese AM. Histological assessment of the teratogenic potential of the pesticide folidol to induce the appearance of cleft palate in wistar rats. Pharmacologyonline. 2006 Ago;2:44-53.

[48] Wattiez C. Links between in utero exposure to pesticides and effects on the human progeny. Does European pesticide policy protect health? Congenital Diseases and the Environment. 2007;23:183-206.

[49] Restrepo M, Muñoz N, Day N, Parra JE, Hernández C, Blettner M, et al. Birth defects among children born to a population occupationally exposed to pesticides in Colombia. Scand J Work Environ Health. 1990;16(4):239-246.

[50] Muñoz J, Bustos I, Quintero C y Giraldo A. Factores de Riesgo para Algunas Anomalías Congénitas en Población Colombiana. Rev. Salud Pública. 2001 Sept;3(3): 268-82. 